\title{
AN ARITHMETIC MEAN OF INFORMATION GAIN AND CORRELATION RATIO BASED DECISION TREE ALGORITHM FOR ACCIDENT DATASET MINING: A CASE STUDY OF ACCIDENT DATASET OF GOMBE - NUMAN -YOLA HIGH WAY, NIGERIA
}

\author{
B. Z. Yahaya $^{1 *}$, L. J. Muhammad ${ }^{2}$, N. Abdulganiyyi ${ }^{3}$, F. S. Ishaq ${ }^{4}$ and Y. Atomsa ${ }^{5}$ \\ 1,2,3,4,5 Mathematics \& Computer Science Department, Federal University of \\ Kashere, Gombe State, Nigeria \\ 1'baladada57@yahoo.com, ${ }^{2}$ lawan.jibril@ fukashere.edu.ng, ${ }^{3}$ agnuraini@gmail.com, \\ ${ }^{4}$ faisalsishaq@gmail.com, 5 au.nlaro@gmail.com
}

\begin{abstract}
Road traffic accident datasets have large number of attributes with different data types and district values. As such applying information gain based decision tree data mining algorithms would not give good accuracy which may affect hidden patterns or useful knowledge to uncover from the dataset. This study proposed An Arithmetic Mean of Information Gain and Correlation Ratio Based Decision Tree data mining algorithm which addressed the biasness and improve the accuracy of Information Gain Based Decision tree data mining algorithms. The proposed algorithm was demonstrated using road accident dataset of Gombe Numan -Yola High Way, Nigeria and gave 93.29 \% accuracy against Information Gain Decision tree algorithm which gave $\mathbf{7 4 . 9 3 \%}$ accuracy. The proposed algorithm minimized the biasness disadvantage of the information Gain of decision tree based algorithm for datasets with large number of attributes with different data types and district values.
\end{abstract}

Keywords - Data mining, algorithm, information gain, correlation ratio, arithmetic mean, dataset

\section{INTRODUCTION}

Road Traffic Accidents killed more than 1.2 million people, and injured between 20 and 50 million others in 2004, thereby becoming the ninth most common cause of death in that year and remains among the most central public health problems in the world [9]. Huge amount data are generating due to frequent road traffic accidents in Nigeria [13][14]. Those dataset are available in both unstructured and structured forms [11][10]. There are data mining algorithms such as Decision Tree algorithm[1][2],Naïve Byers[3][4],Neural Network Model [1],[2],Support Vector Machine[2],[5][12] are used to uncover hidden patterns or useful knowledge. The decision trees are widely used for classification and prediction. The well-known decision tree algorithms are ID3 and C4.5. The ID3 uses information Gain as the splitting criterion for an attribute. The attribute with highest Information Gain (IG) is considered as the splitting attribute. The information Gain has a disadvantage such as biasness toward the attribute with larger number of district values. As a result, IG based method is not effective for all type of dataset.

Received: March 5, 2019

Reviewed: May 29, 2019

Accepted: June 2, 2019

* Corresponding Author

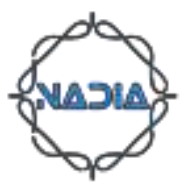


For instance, if a dataset has deferent attribute with deferent number of distinct values, the IG based method will prefer the attribute which has more number of distinct values as the splitting attribute despite the fact that other attribute with less number of distinct values may be more relevant for classification. Thus, IG based method has a drawback. To resolve this problem, we have proposed an Arithmetic Mean of Information Gain and Correlation Ratio decision tree model. This approach is applicable to all type of datasets.

\section{RELATED WORK}

[6] Proposed a Correlation Ratio Based Decision Tree Model for Health Care Data Mining which is a variant of decision tree model. This approach uses the concept of correlation Ratio.

[7] Proposed a new splitting criterion based on the classical foreword selection method. In this approach, the variable having the largest absolute correlation with target value is choosing as the best splitting variable in each node and the Support Vector Machine SVM is used to find the best threshold on the selected variable to classify the data. This approach produced a decision tree that has a shorter high than the previous approach.

[8] Proposed an improved C4.5 based on a backsword adjustment strategy and optimization of the $\mathrm{C} 4.5$ parameters. In this approach, additional phase was interjected between the growing and the pruning phases of the C4.5 classifier and also, C4.5 parameters such as CF and MS were optimized. This approach allows user to obtain a decision tree with high merit such as size and accuracy than the default C4.5.

C4.5 data mining algorithm was scaling up using $L$ hospital rule by removing all the logarithms and antilogarithms in its calculation process for mining large dataset in [15]. L' hospital rule was employed in order to improve the traditional $\mathrm{C} 4.5$ algorithm where the average of the information gain ratio and information gain was used. The study shows that, the improved $\mathrm{C} 4.5$ algorithm has the best running time of $\mathrm{O}(\mathrm{n})$ compared to traditional $\mathrm{C} 4.5$ algorithm which has $\mathrm{O}(\mathrm{n}(\log 2 \mathrm{n}) 2)$ and proved to be more efficient compared to $\mathrm{C} 4.5$ algorithm when the dataset is large. Nevertheless, for small dataset is traditional C4.5 is more efficient.

\section{ARITHMETIC MEAN OF INFORMATION GAIN AND CORRELATION RATIO BASED DECISION TREE}

\subsection{OVERVIEW OF CORRELATION RATIO}

The Correlation Ratio method is more robust than the correlation coefficient this is because the later is more suitable to only for application with quantitative outcome. The CR method can be used to partition the sample into different categories according to the observe outcome. An attribute is said to be significant if it can identify at least one outcome class where the average value of the attribute and the average on all classes as different. Suppose that there is a set of $\alpha$ turples in a dataset D. let the number of times that outcome y belong to $\mathrm{Y}$ is $\alpha_{\mathrm{y}}$ so that the dataset can be partitioned by their outcome as follows

$$
\forall y \in Y \mid S_{y}=\left(x_{j y}^{(1)}, \ldots, x_{j y}^{(n)} j=1 \ldots \alpha_{y}\right.
$$

Where $S_{\mathrm{y}}$ is the set of all turples with outcome $\mathrm{y}$ and $x_{j y}^{(1)}$ is the value of the ith attribute of the jth tuple among all the $\alpha_{y}$ turples with outcome y.

$$
\forall y \in Y \mid \bar{x}_{y}^{(i)}=\frac{\sum_{j=1}^{\alpha_{y}} x_{j y}^{(1)}}{\alpha_{y}}
$$


The overall average of the ith attribute from all turple can be expressed as

$$
\bar{x}^{(i)}=\frac{\sum_{y \in Y} \alpha_{y} \bar{x}_{y}^{(i)}}{\alpha}
$$

The CR is given by

$$
C r_{i}=\sqrt{\frac{\sum_{y \in Y} \alpha_{y}\left(\bar{x}_{y}^{(0)}-\bar{x}^{(i)}\right)^{2}}{\sum_{j=1}^{\alpha_{y}}\left(x_{j y}^{(j)}-\bar{x}^{(0)}\right)^{2}}}
$$

\subsection{OVERVIEW OF THE INFORMATION GAIN}

Information Gain (IG) measures how much information a feature gives us about the class. Information Gain (IG) in other word measure the reduction in entropy as show below Assuming we have a dataset $\mathrm{D}$ with and attribute A, the entropy of the whole dataset $\mathrm{D}$ is given by

$$
E(D)=(p, n)=-\frac{p}{p+n} \log _{2} \frac{p}{p+n}-\frac{n}{p+n} \log _{2} \frac{n}{p+n}
$$

Where $\mathrm{p}$ is the number of positive, $\mathrm{n}$ is the number of negative

The expected new entropy of an attribute $\mathrm{A}$ is given by

$$
E(A)=\sum_{j=1}^{r} \frac{p_{j}+n_{j}}{p+n} I(p, n)
$$

Therefore, the Information Gain of attribute A is given by

$$
I G(A)=E(D)-E(A)
$$

\subsection{ILLUSTRATION OF ARITHMETIC MEAN OF INFORMATION GAIN AND CORRELATION RATIO (AMIGCR) COMPUTATION}

Below is the illustration of the computation of AMIGCR using weather dataset in Table I-IV .

Table I. Outlook

\begin{tabular}{|c|c|c|c|}
\hline Class & Overcast & Rainy & Sunny \\
\hline Yes & 4 & 3 & 2 \\
\hline No & 2 & 2 & 3 \\
\hline
\end{tabular}

$$
\begin{aligned}
& x_{\text {yes }}^{(1)}=\frac{4}{9}=0.44, \quad x_{\text {no }}^{(1)}=\frac{3}{5}=0.6, \quad \bar{x}^{(1)}=\frac{7}{14}=0.5 \\
& C r_{\text {Outlook }}=\sqrt{\frac{5(0.6-0.5)^{2}+9(0.44-0.5)^{2}}{(0-0.5)^{2}+(2-0.5)^{2}+(3-0.5)^{2}+(4-0.5)^{2}+(3-0.5)^{2}+(2-0.5)^{2}}}=0.053 \\
& I G_{\text {Outlook }}=0.25 \\
& \text { AMIGCR } R_{\text {Outlook }}=\frac{(0.053+0.25)}{2}=\mathbf{0 . 1 5 2}
\end{aligned}
$$

Table II. Temperature

\begin{tabular}{|c|c|c|c|}
\hline Class & Cool & Rainy & Sunny \\
\hline Yes & 3 & 2 & 4 \\
\hline No & 1 & 2 & 2 \\
\hline
\end{tabular}




$$
\begin{aligned}
& x_{\text {yes }}^{(2)}=\frac{4}{9}=0.44, \quad x_{n o}^{(2)}=\frac{2}{5}=0.4, \quad \bar{x}^{(2)}=6 / 14=0.428 \\
& C r_{T \text { emp }}=\sqrt{\frac{9(0.44-0.428)^{2}+5(0.4-0.428)^{2}}{(3-0.428)^{2}+(2-0.428)^{2}+(4-0.428)^{2}+(1-0.428)^{2}+(2-0.428)^{2}+(2-0.428)^{2}}}= \\
& 0.0058 \\
& I G_{\text {Temperature }}=0.03 \\
& A M I G C R_{\text {Humidity }}=\frac{(0.0058+0.03)}{2}=\mathbf{0 . 0 1 8}
\end{aligned}
$$

Table III. Humidity

\begin{tabular}{|l|l|l|}
\hline Class & Normal & High \\
\hline Yes & 6 & 2 \\
\hline No & 1 & 5 \\
\hline
\end{tabular}

$\mathrm{x}_{\text {yes }}^{3}=\frac{6}{8}=0.75, \mathrm{x}_{\text {no }}^{3}=\frac{5}{6}=0.833, \bar{x}^{(3)}=11 / 14=0.785$,

$\mathrm{Cr}$

$$
\begin{gathered}
C r_{\text {Humidity }}=\sqrt{\frac{8(0.75-0.785)^{2}+6(0.833-0.785)^{2}}{(6-0.785)^{2}+(2-0.785)^{2}+(1-0.785)^{2}+(5-0.785)^{2}}} \\
C r_{\text {Humidity }}=0.023 \\
I G_{\text {Humidity }}=0.22 \\
\text { AMIGCR } R_{\text {Humidity }}=\frac{0.023+0.22}{2}=\mathbf{0 . 1 2 2}
\end{gathered}
$$

Table IV. Windy

\begin{tabular}{|l|l|l|}
\hline Class & True & False \\
\hline Yes & 6 & 3 \\
\hline No & 2 & 2 \\
\hline
\end{tabular}

$x_{\text {yes }}^{(4)}=\frac{6}{9}=0.666, \quad x_{\text {no }}^{(4)}=\frac{2}{4}=0.5, \quad \bar{x}^{(4)}=\frac{8}{13}=0.5$

$C r_{\text {Windy }}=\sqrt{\frac{9(0.666-0.615)^{2}+4(0.5-0.615)^{2}}{(6-0.615)^{2}+(3-0.615)^{2}+(2-0.615)^{2}+(2-0.615)^{2}}}=0.044$

$I G_{\text {Windy }}=0.07$

$A M I G C R_{\text {Windy }}=\frac{(0.044+0.07)}{2}=0.057$

ConstructingArithmetic Mean of Information Gain and Correlation Ratio Based Decision Tree

1. //Input: Dataset D and Node N

2. Let $A=A_{1}, A_{2}, \ldots A_{n}$ denote the set of $n$ attribute for the turple in $D$

3. If all the turples in $\mathrm{D}$ have the same class label Then

4. Return $\mathrm{N}$ as a leaf node labeled with the class label

5. Else 
6. For each attribute $A_{i}$ Do.

7. $\mathrm{Cr}_{\mathrm{i}}=$ Correlation-ratio $\left(\mathrm{A}_{\mathrm{i}}, \mathrm{Y}\right)$ where $\mathrm{Y}$ is the class attribute

8. Insert $\mathrm{Cr}_{\mathrm{i}}$ in set $\mathrm{CR}$, where, $\mathrm{CR}$ is the set of correlation ratio

9. $\mathrm{IG}_{\mathrm{i}}=$ Information Gain $\left(\mathrm{A}_{\mathrm{i}}, \mathrm{Y}\right)($ fomular 6$)$

10. $\operatorname{AMIGCR}_{\mathrm{i}}\left(\mathrm{A}_{\mathrm{i}} \mathrm{Y}\right)=\left(\mathrm{Cr}_{\mathrm{i}}+\mathrm{IG}_{\mathrm{i}}\right) / 2$, where, AIGCR is an Arithmetic Mean of Information Gain and Corelation ratio

11. End For

12. $r=\max ($ AMIGCR $)$

13. If multiple $A_{i}$ have $A I G C R==r$ Then

14. Choose the attribute $A_{i}$ as the splitting attribute which has most of the possible distinct values present in $\mathrm{D}$

15. Else

16. Choose the attribute $A_{i}$ as the splitting attribute whose $A_{I G C R}==r$

17. End If

18. Label node $\mathrm{N}$ with attribute $\mathrm{A}_{\mathrm{i}}$

19. let attribute $A_{i}$ has $m$ distinct values $A_{i 1}, A_{i 2}, \ldots A_{i m}$

20. Divide $D$ into partitions $D=D_{1}, D_{2}, \ldots D_{m}$ corresponding to each distinct value of $\mathrm{A}_{\mathrm{i}}$ respectively, and create a child node $\mathrm{N}_{\mathrm{j}}$ corresponding to each partition from node $\mathrm{N}$ with corresponding distinct value of attribute $\mathrm{A}_{\mathrm{i}}$ as the label on the branch For each partition $D_{j}$ in D Do

21. If the partition is empty Then

22. Label node $\mathrm{N}_{\mathrm{j}}$ as a leaf node with majority class in $\mathrm{D}$

23. Else

24. Call Decision Tree $\left(D_{j}, N_{j}\right)$

25. End If

26. End If

\section{End If}

\section{EXPERIMENT AND RESULT}

\subsection{ACCIDENT DATASET OF GOMBE-NUMAN-YOLA HIGH WAY}

The study used data set of traffic road accident for Gombe-Numan-Yola High Way in Nigeria. Gombe-Numan-Yola High Way is the road that linked Gombe State to Adamawa State in Nigeria and its distance is $251 \mathrm{Km}$. the road is dilapidated and there are frequency of road accidents along it which claims many lives and properties worth several millions of naira. Accident Dataset was for the road traffic accident records from January 2000 and June, 2018 of Gombe-NumanYola High Way with seven hundred and fourteen instances. The dataset used to 
valid the proposed Arithmetic Mean of Information Gain and Correlation Ratio decision tree based algorithm against information gain based decision tree algorithm (ID3). The dataset has five attributes which include location of Accident, time of the accident, type of vehicle involved in the accident, number of casualties and cause of the accident. The Table V shows the description, value and data type of each attribute of the dataset.

Table V. Description of Dataset Attributes

\begin{tabular}{|c|c|c|c|c|}
\hline $\mathrm{SN}$ & Attribute & Description & Value & Data type \\
\hline 1 & $\begin{array}{l}\text { Location of the } \\
\text { Accident }\end{array}$ & $\begin{array}{l}\text { LocationI (Gombe }- \text { Kumo) } \\
\text { LocationII (Kumo - Kaltingo) } \\
\text { LocationIII (Kaltingo - Numa) } \\
\text { LocationIV (Numan - Yola) }\end{array}$ & $\begin{array}{l}\text { A } \\
\text { B } \\
\text { C } \\
\text { D }\end{array}$ & $\begin{array}{l}\text { Categorical } \\
\text { Categorical } \\
\text { Categorical } \\
\text { Categorical }\end{array}$ \\
\hline 2 & Time of the Accident & $\begin{array}{l}\text { Morning } \\
\text { Afternoon } \\
\text { Evening }\end{array}$ & $\begin{array}{l}\text { A } \\
\text { B } \\
\text { C }\end{array}$ & $\begin{array}{l}\text { Categorical } \\
\text { Categorical } \\
\text { Categorical }\end{array}$ \\
\hline 3 & Type of Vehicle & $\begin{array}{l}\text { Light Car } \\
\text { Heavy Car } \\
\text { Light Car \& Heavy Car }\end{array}$ & $\begin{array}{l}\mathrm{A} \\
\mathrm{B} \\
\mathrm{C}\end{array}$ & $\begin{array}{l}\text { Categorical } \\
\text { Categorical } \\
\text { Categorical }\end{array}$ \\
\hline 4 & Number of Causalities & numeric & numeric & numeric \\
\hline 5 & Cause of the Accident & $\begin{array}{l}\text { DistractedDriving } \\
\text { BrakeFailure } \\
\text { OverSpeed } \\
\text { UncertainCause } \\
\text { DrunkDriving } \\
\text { RunningStopSigns } \\
\text { TeenageDrivers } \\
\text { NightDriving } \\
\text { ImproperTurns } \\
\text { Potholes } \\
\text { DrowsyDriving } \\
\text { TireBlowouts } \\
\text { DeadlyCurves } \\
\text { AnimalCrossings } \\
\text { OverTaking }\end{array}$ & $\begin{array}{l}\text { A } \\
B \\
\text { C } \\
\text { D } \\
\text { E } \\
\text { F } \\
\text { G } \\
\text { H } \\
\text { I } \\
\text { J } \\
\text { K } \\
\text { L } \\
\text { M } \\
N \\
O\end{array}$ & $\begin{array}{l}\text { Categorical } \\
\text { Categorical } \\
\text { Categorical } \\
\text { Categorical } \\
\text { Categorical } \\
\text { Categorical } \\
\text { Categorical } \\
\text { Categorical } \\
\text { Categorical } \\
\text { Categorical } \\
\text { Categorical } \\
\text { Categorical } \\
\text { Categorical } \\
\text { Categorical } \\
\text { Categorical }\end{array}$ \\
\hline
\end{tabular}

\subsection{RESULT AND DISCUSSION}

Weka data mining software was used for the experiment of the dataset of the study. Weka open source data mining software was used to mine the dataset. Weka contains machine learning algorithms for data mining tasks. The algorithm can either be called to java code or apply directly to a dataset. The proposed Arithmetic Mean of Information Gain and Correlation Ratio based decision tree algorithm was encoded into the weka so as to determine to its accuracy of the proposed algorithm against Information Gain decision tree based algorithm (ID3) which is one of the available algorithms in Weka. Accuracy is one of the data mining evaluation mechanisms which measure the proportion of the total number of predictions that were correctly predicted by an algorithm.

$$
\text { Accuracy }=\frac{t p+t n}{t p+t n+f p+f n}
$$

The result of the experiments showed that Arithmetic Mean of Information Gain and Correlation Ratio Based Decision Tree algorithm has $93.29 \%$ accuracy against Information Gain Decision tree algorithm which has $74.93 \%$ accuracy. The result of the experiment is shown in Table VI.

VI. Experimental Results 


\begin{tabular}{|l|l|l|}
\hline Decision tree algorithm & $\begin{array}{l}\text { AMIGCR Based Decision Tree } \\
\text { Algorithm }\end{array}$ & $\begin{array}{l}\text { IG Based Decision Tree } \\
\text { Algorithm (ID3) }\end{array}$ \\
\hline Accuracy & $93.29 \%$ & $74.93 \%$ \\
\hline
\end{tabular}

From the experimental result of the analysis, our proposed Arithmetic Mean of Information Gain and Correlation Ratio based decision tree algorithm has minimize the biasness disadvantage of the information Gain Decision Tree Algorithm (ID3) for the attribute with larger number of district values. Thus, our proposed algorithm address the drawback of Information Gain Decision Tree algorithm which prefers attributes with more than distinct values rather than attribute with less than number of distinct values.

\section{CONCLUSION}

There are many dataset for accident and each dataset has its own nature thus, varies in nature and distribution patterns. However, those datasets generally have large number of attributes with different data types and district values. Therefore, harnessing information gains decision tree data mining algorithms may not be give the good accuracy which may affect hidden patterns or useful knowledge to uncover from the dataset. Therefore, the proposed Arithmetic Mean of Information Gain and Correlation Ratio Based Decision Tree data mining algorithm address the biasness and improve the accuracy of Information Gain Based Decision tree data mining algorithm which it was demonstrated using accident dataset of Gombe - Numan -Yola High Way, Nigeria. In the future the proposed algorithm would be applied to other non-accidental dataset such healthcare dataset.

\section{ACKNOWLEDGMENT}

This work was supported by the Tertiary Education Trust Fund, Nigeria (TETFUND), as an Institution Based Research Fund (IBR) for Federal University of Kashere, Gombe State, Nigeria.

\section{REFERENCES}

[1] Murata, N., Yoshizawa S. and Amari, S. I. Network Information Criterion-detemining the Number of Hidding Units for an Artificial Neural Network Model, 5, 1994, pp 865-872

[2] Han, J., Kamber, M. and Pei, J. "Data Mining Concept and Techniques". Morgan Koufman, 2006

[3] John G. H. and Langley, P. "Estimating Continuous Distribution in Beryesian Classifier", 1995, pp.338345.

[4] Domingos, P. and Pazzani, M. "On the Optimality of the Simple Bayesian Classifier Under Zero -one Loss", 2002, pp. 103-130.

[5] Vapnik, M. V. "The Nature of Statistical Learning Theory". Springer, 1995

[6] Roy, S., Mondal, S., Ekbal, A. and Desarkar, M. S. CRDT: Correlation Ratio Based Decision Tree Model for Healthcare Data Mining, 2016 IEEE $16^{\text {th }}$ International Conference on Bioinformatics and Bioengineering (BIBE), Taichung, Taiwan, 2016, pp. 36-43

[7] Hadi, S. Y. and Nima, S. "Multi Brach Decision Tree: a New Splitting Criterion", International Journal of Advance Science and Technology. 45, 2012, pp.91-106.

[8] Beck, J. R., Garcia, M. G. and Anagnostopoulos, G.C “A Backward Adjustng Strategy and Optimization of the C4.5 Parameters to improve C4.5's Performance" Proceeding of the Twenty-First FLAIRS Conference, 2008

[9] Muhammad, L. J. Sani, S., Yakubu, A., Yusuf, M. M. and Elrufai, T. A, Mohammed, I. A. and. Nuhu, A. M. "Using Decision Tree Data Mining Algorithm to Predict Causes of Road Traffic Accidents, its Prone Locations and Time along Kano -Wudil Highway", International Journal of Database Theory and Application, 10, 2016, pp. 197-206.

[10] Muhammad, L. J., Yakubu, A. and Mohammed, I. A. 'Data Mining Driven Approach for Predicting Causes of Road Accident', 13th International Conference 2017- Information Technology for Sustainable Development, Nigeria Computer Society, 28, 2017, pp. 10-15

[11] Muhammad, L. J., Mohammed, I. A. and Yakubu, A. "Social Media Analytics Driven Counterterrorism Tool to improve Intelligence Gathering towards Combating Terrorism in Nigeria" International Journal of Advanced Science and Technology, 107, 2017, pp.33-42 
[12] Muhammad, L. J., Garba, E. J., Oye N. D. and Wajiga, G. M "On the Problems of Knowledge Acquisition and Representation of Expert System for Diagnosis of Coronary Artery Disease (CAD)", International Journal of u- and e- Service, Science and Technology, 11:3, 2018, pp. 49-58.

[13] Hussain, S., Muhammad, L. J., Atomsa, Y., Mining Social Media and DBpedia Data Using Gephi and R, Journal of Applied Computer Science \& Mathematics, 12:1, 2018, pp.15-20

[14] Hussain, S., Muhammad, L. J., Atomsa, Y. and Mohammed I. A., Performance Evaluation of Various Data Mining Algorithms on Road Traffic Accident Dataset, Information and Communication Technology for Intelligent Systems Proceedings of ICTIS, 106(1), 2018.

[15] Yahaya, B. Z, Muhammad, L. J, Abdulganiyyu, N., Ishaq F. S and Atomsa, Y. An Improved C4.5 Algorithm Using L'Hospital Rule for Large Dataset, Indian Journal of Science and Technology, 11:47, 2018. 